\title{
Developmental Toxicity of Thyroid-Active Compounds in a Zebrafish Embryotoxicity Test
}

Barae Jomaa $^{1}$, Sanne A. B. Hermsen ${ }^{2}$, Maurijn Y. Kessels ${ }^{3,4}$, Johannes H. J. van den Berg ${ }^{1}$, Ad A.C.M. Peijnenburg ${ }^{5}$, Jac M.M.J. G. Aarts ${ }^{1}$, Aldert H. Piersma ${ }^{2}$ and Ivonne M. C. M. Rietjens ${ }^{1}$ ${ }^{1}$ Division of Toxicology, Wageningen University, Wageningen, The Netherlands; ${ }^{2}$ Laboratory for Health Protection Research, National Institute for Public Health and the Environment (RIVM), Bilthoven, The Netherlands; ${ }^{3}$ Department of Biochemistry, Wageningen University, Wageningen, The Netherlands; ${ }^{4}$ Experimental Zoology Group, Wageningen University, Wageningen, The Netherlands; ${ }^{5}$ RIKILT - Institute of Food Safety, Wageningen, The Netherlands

\section{Summary}

Zebrafish embryos were exposed to concentration ranges of selected thyroid-active model compounds in order to assess the applicability of zebrafish-based developmental scoring systems within an alternative testing strategy to detect the developmental toxicity of thyroid-active compounds. Model compounds tested included triiodothyronine (T3), propylthiouracil (PTU), methimazole (MMI), sodium perchlorate $\left(\mathrm{NaClO}_{4}\right)$ and amiodarone hydrochloride (AMI), selected to represent different modes of action affecting thyroid activity. Tested time windows included 48-120 hours post fertilization (hpf), 0-72 hpf and 0-120 hpf. All tested compounds resulted in developmental changes, with T3 being the most potent. The developmental parameters affected included reflective iridophores, beat and glide swimming, inflated swim bladders, as well as resorbed yolk sacs. These effects are only evident by $120 \mathrm{hpf}$ and therefore an existing General Morphology Score (GMS) system was extended to create a General Developmental Score (GDS) that extends beyond the $72 \mathrm{hpf}$ scoring limit of GMS and includes additional parameters that are affected by exposure to model thyroid-active compounds. Moreover, the GDS is cumulative as it includes not only the scoring of developmental morphologies but also integrates developmental dysmorphologies.

Exposures from 48-120 hpf did not provide additional information to exposures from 0-120 hpf. The results indicate that the zebrafish GDS can detect the developmental toxicity of thyroid toxicants and may be of use in an integrated testing strategy to reduce, refine and, in certain cases, replace animal testing.

Keywords: thyroid, zebrafish, developmental toxicity, morphological scoring, in vitro alternatives

\section{Introduction}

The EU Regulation on the Registration, Evaluation, Authorisation and Restriction of Chemicals (REACH) requires all substances marketed or manufactured in quantities above 10 tons per annum to be tested for developmental toxicity (OECD 421 or OECD 422). Substances that are marketed or manufactured in quantities above 100 tons per annum are required to be tested for pre-natal developmental toxicity (OECD 414) (REACH, 2006). Based on data on chemical production from 1991 to 1994, the EU estimates that 2.6 million animals will be needed for all REACH testing or a total of 9 million when offspring produced during reproductive and developmental toxicity test- ing are taken into account. While some studies have suggested much higher numbers, the EU is sticking to its initial predictions (ECHA, 2009; Hartung and Rovida, 2009; van der Jagt et al., 2004; Pedersen et al., 2003). The issues associated with these tests include ethical acceptability considerations, high costs and long duration. This has prompted research into the development of suitable high-throughput alternative tests that, once validated, could reduce animal testing within an integrated testing strategy (ITS).

In a previous study we investigated to what extent thyroid (TSH-screen) and pituitary (T-screen) cell proliferation assays could be used as in vitro screens for detecting the effects of thyroid hormone-disrupting compounds on thyroid and pituitary

Received February 1, 2014; accepted in revised form April 7, 2014; Epub April 10, 2014;

http://dx.doi.org/10.14573/altex.1402011

Abbreviations: AMI, amiodarone hydrochloride; dpf, days post-fertilization; hpf, hours post-fertilization; $\mathrm{EC}_{50}$, effective concentration $50 \%$; GDS, General Development Score; GMS, General Morphology Score; HPT axis, hypothalamus-pituitary-thyroid axis; IC $_{50}$, inhibitory concentration 50\%; IHC, immunohistochemistry; ITS, integrated testing strategy; MMI, methimazole; PTU, propylthiouracil;

T3, triiodothyronine; T4, tetraiodothyronine; $\mathrm{TH}$, thyroid hormone 
weights used as endpoints in in vivo assays (Jomaa et al., 2013). While the effect of estrogen-mimicking compounds on cell proliferation in the in vitro E-screen was found to correlate with their effects on in vivo uterine weight $\left(\mathrm{r}^{2}=0.85\right)$ (Wang et al., 2012), the effects of thyroid-active compounds on thyroid (TSHscreen) and pituitary (T-screen) cell proliferation was found to have poor correlation with their effects on the weight of these respective organs in vivo. The results of the study indicated that a complex interplay between factors within the hypothalamuspituitary-thyroid (HPT) axis may underlie the effects of thyroidactive compounds on thyroid and pituitary organ weight endpoints in vivo. Therefore, it was proposed that the development of future alternative tests, aiming at the refinement, reduction, or replacement of animal studies, should include as an initial screen a broad battery of in vitro tests that cover the various modes of action of thyroid-active compounds and should in addition be based on more complex model systems that better integrate the various processes involved in the HPT axis. The vertebrate Danio rerio (zebrafish) may represent such a complex model system that includes an intact HPT axis. Given that alterations in the thyroid system can have severe effects on development (Boyages and Halpern, 1993; Haddow et al., 1999; Utiger, 1999), the aim of the present study was to investigate whether a zebrafish-based developmental toxicity assay would be able to detect thyroid hormone-active compounds.

Hermsen et al. (2011) developed a zebrafish-based quantitative scoring system for developmental and teratogenic endpoints, with the former called the general morphology score (GMS). The developmental endpoints include abnormalities related to the completion of gastrulation, formation of somites, development of the eyes, spontaneous movement, blood circulation, pigmentation, edemata, malformations of the chorda structure, spinal cord (scoliosis, rachitis), head, sacculi/otoliths, tail, heart, yolk sac, growth retardation, and tail length (Nagel, 2002). The GMS is meant to be semi-quantitative and hence more efficient than more complex scoring systems while maintaining the same relevance in terms of output (Hermsen et al., 2011). The GMS scoring system uses the 0-72 hpf time window and was vali- dated using eight glycol ethers and six 1,2,4-triazole antifungals (Hermsen et al., 2011). While alternative methods for developmental toxicity testing are unlikely to replace testing on rodents, they can potentially be used in pre-screening in order to reduce the need for consecutive in vivo testing (Piersma, 2006).

In order to investigate whether a zebrafish-based developmental toxicity assay would be able to detect thyroid hormoneactive compounds, the effects in the zebrafish-based bioassay of a series of model thyroid-active compounds representing different modes of thyroid action in vivo (Tab. 1) was investigated. The selected compounds were triiodothyronine (T3), sodium perchlorate $\left(\mathrm{NaClO}_{4}\right)$, propylthiouracil (PTU), methimazole (MMI) and amiodarone (AMI). All selected compounds are known to affect thyroid hormone levels in vivo albeit by different modes of action (Grover et al., 2007; Hood et al., 1999; de Sandro et al., 1991; Stoker et al., 2006; Yamasaki et al., 2002).

\section{Materials and methods}

\section{Compounds}

All compounds were obtained from Sigma-Aldrich Chemie (Zwijndrecht, The Netherlands) and were of high purity ( $\geq 95 \%)$. All test chemicals were added from 500-fold concentrated stock solutions in dimethylsulfoxide (DMSO, Acros Organics, Geel, Belgium) to Dutch Standard Water (DSW; demineralised water supplemented with $\mathrm{NaHCO}_{3}(100 \mathrm{mg} / \mathrm{l}), \mathrm{CaCl}_{2} \cdot \mathrm{H}_{2} \mathrm{O}(200 \mathrm{mg} / \mathrm{l})$, $\mathrm{KHCO}_{3}(20 \mathrm{mg} / \mathrm{l})$, and $\mathrm{MgSO}_{4} \cdot 7 \mathrm{H}_{2} \mathrm{O}(180 \mathrm{mg} / \mathrm{l})$ ). The exposure medium was then aerated for $24 \mathrm{~h}$ at $27^{\circ} \mathrm{C}$, the $\mathrm{pH}$ was adjusted to 7.4-8.4 and $\mathrm{O}_{2}$ concentration was above $6.5 \mathrm{mg} / \mathrm{l}$. The concentration range that was selected for the model compounds was based on reported effective concentrations for inducing reduction in T4 levels in zebrafish (Raldúa and Babin, 2009).

\section{Zebrafish embryos}

Zebrafish, obtained commercially (Ruinemans Aquarium BV, Montfoort, The Netherlands), were maintained and bred at RIVM (Bilthoven, The Netherlands) for more than five years.

\section{Tab. 1: Selected model thyroid-active compounds}

List of compounds used in this study with their main mode of action on the thyroid hormone system based on rodent studies, and concentration ranges used in the experiments. The logarithm of the octanol/water partition coefficient (logP), which is a measure of lipophilicity, was predicted based on the chemical structure of the test compound using chemaxon software.

\begin{tabular}{|l|l|l|l|l|l|l|}
\hline Compound & Abbreviation & CAS No. & Description & logP & Mode of Action & $\begin{array}{l}\text { Concentration } \\
\text { Range }(\mu \mathrm{M})\end{array}$ \\
\hline Triiodothyronine & T3 & $6893-02-3$ & Endogenous ligand & 2.80 & $\begin{array}{l}\text { Thyroid hormone } \\
\text { receptor agonist }\end{array}$ & $0.00005-0.5$ \\
\hline Propylthiouracil & PTU & $51-52-5$ & Antithyroid drug & 1.2 & $\begin{array}{l}\text { Inhibits thyroid peroxidase } \\
\text { and deiodinase type } 1\end{array}$ & $25-400$ \\
\hline Methimazole & $\mathrm{MMI}$ & $60-56-0$ & Antithyroid drug & 0.75 & Inhibits thyroid peroxidase & $125-2,000$ \\
\hline $\begin{array}{l}\text { Sodium perchlorate } \\
\text { monohydrate }\end{array}$ & $\mathrm{NaClO} \cdot \mathrm{H}_{2} \mathrm{O}$ & $7791-07-3$ & $\begin{array}{l}\text { Environmental } \\
\text { contaminant }\end{array}$ & -0.10 & Inhibits iodide uptake & $25-400$ \\
\hline $\begin{array}{l}\text { Amiodarone } \\
\text { hydrochloride }\end{array}$ & $\mathrm{AMI}$ & $19774-82-4$ & Antiarrhythmic drug & 7.64 & Increases iodine load & $0.001-10$ \\
\hline
\end{tabular}


Their daily diet consisted of two servings of dry flakes (Special Diet Services, Tecnilab-BMI BV, The Netherlands) and one serving of Artemia (Landman BV, The Netherlands). Constant filtering and permanent water flow-through kept contaminants to a minimum. Male and female adult zebrafish free from externally visible diseases were housed together in 7.5 1 ZebTEC aquaria at $27^{\circ} \mathrm{C} \pm 1^{\circ} \mathrm{C}$ with a light/dark (LD) cycle of 14-hour light/10-hour dark. Three days prior to mating, males and females were placed in spawning chambers within separate tanks. Mating involved placing two males with two females in the spawning chambers with reduced water flow and keeping them in the dark overnight after which turning on the lights induced mating, spawning and fertilization within 30 minutes. The spawning chambers have a perforated bottom surface that allows the eggs to fall through, thus preventing the predation of eggs by adult zebrafish.

\section{Exposure}

Spawned eggs were rinsed in DSW and unfertilized eggs, less than $10 \%$ of total, were removed. Embryos were selected at the 4 to 32-cell stage and one embryo was added per well of a 24-well plate containing $2 \mathrm{ml}$ of test medium per well. Alternatively, the transfer to the 24-well plates containing $2 \mathrm{ml}$ of the test medium was performed at $48 \mathrm{hpf}$. The 24 -well plates were kept in an incubator at $26.5^{\circ} \mathrm{C} \pm 1^{\circ} \mathrm{C}$ with a light/dark (LD) cycle of 14-hour light/10-hour dark. Exposure was performed at the start of the light cycle.

\section{General morphology score (GMS)}

Scoring of the developmental morphology was performed using a Leica Labovert FS microscope as outlined by Hermsen et al. (2011). Briefly, an incremental binary scoring system was used to assign a 0 to a non-event and a 1 to each normal morphologic developmental event that is easy to visualize under a regular stereo microscope. Sets of developmental events included detachment of tail, somite formation, eye development, movement, heartbeat, blood circulation, pigmentation of the head and body, pigmentation of the tail, pectoral fin development, protruding mouth, and hatching. The subtotals of all sets of developmental events were added towards a general morphology score (GMS), which in normal non-exposed embryos amounts to 15 at the 72 hour time point. In a separate binary scoring system, teratogenicity was scored, whereby normal embryos were assigned a 1 and malformed embryos were given a 0 for every malformation studied. These malformations included pericardial edema, yolk sac edema, eye edema, malformation of the head, absence/malformation of sacculi/otoliths, malformation of tail, malformation of heart, modified chorda structure, scoliosis, rachischisis and yolk deformation. Testing was considered valid when no more than $10 \%$ of control embryos were coagulated, underdeveloped or malformed.

\section{Immunohistochemistry}

Whole-mount immunohistochemistry of T4 was performed as previously described by Raldúa and Babin (2009), with slight modifications. Briefly, zebrafish embryos at $5 \mathrm{dpf}$ were sacri- ficed by incubation on ice for 5 min and then fixed overnight in $4 \%$ paraformaldehyde at $4^{\circ} \mathrm{C}$. They were then washed repeatedly with PBS, dehydrated with a methanol row (30\%, $60 \%, 100 \%)$ and stored overnight or longer at $-20^{\circ} \mathrm{C}$. The embryos were then rehydrated with a methanol row $(60 \%$, $30 \%$ ) and washed several times with PBS containing 1\% Triton X-100. Permeabilization was achieved by 15 minute incubation at room temperature with $0.1 \%$ collagenase in PBS. Immediately after the incubation, the embryos were washed several times with PBS containing 1\% Triton X-100 and blocked in blocking buffer containing $4 \%$ goat serum, $1 \%$ BSA, $1 \%$ DMSO, $0.8 \%$ Triton X-100 and $0.1 \%$ Tween-20 in PBS for $2 \mathrm{~h}$. This was followed by two washes in 1\% BSA in PBS and an overnight incubation in a solution consisting of a polyclonal antibody against T4 (MP Biochemicals, Amsterdam, The Netherlands) diluted 1:4,000 in blocking buffer containing $0.02 \%$ sodium azide. The following day, the embryos were washed several times in $1 \%$ BSA in PBS and incubated on a gentle rotation device for $2 \mathrm{~h}$ in a solution containing a 1:300 dilution of the secondary antibody conjugated to the fluorescent label Alexa Fluor 488 (Life Technologies, Breda, The Netherlands). Right after the incubation, the embryos were either stored in glycerol or embedded in $1 \%$ gelatine for imaging on an a Zeiss Axiovert $100 \mathrm{M}$ confocal microscope (Zeiss Netherlands bv, Weesp, The Netherlands).

\section{Data analysis}

Non-linear curve fitting was done using the Hill equation with the help of GraphPad Prism software version 5.04 (GraphPad Software, San Diego, CA, USA). For the in vivo data that were collected from literature, a dose of $10 \mathrm{ppm} \mathrm{MMI,} \mathrm{equivalent} \mathrm{to} 10$ $\mathrm{mg} / \mathrm{kg}$, was converted to $0.5 \mathrm{mg} / \mathrm{kg}$ bw/day based on the assumption that rats consume $5 \%$ of their body weight of food each day.

\section{Results}

\subsection{Zebrafish thyroid development within selected exposure period}

A limit of exposure was set at $120 \mathrm{hpf}$ (the end of the embryonic phase), which is the regulatory limit to in vitro experimentation as defined by Directive 86/609/EEC (European Council, 1986). To place the exposure regimen into perspective, an analysis of zebrafish, rat and human developmental staging was performed (Fig. 1a). This comparison was based on O'Rahilly and Müller's developmental stages of human embryos, Witschi's development of the rat, and Kimmel's stages of embryonic development of the zebrafish (Kimmel et al., 1995; O'Rahilly and Müller, 1987; Witschi, 1962). Carnegie stage 24 is the last stage of embryonic development and occurs at 33 days for humans, the equivalent of 16 days for rat and $72 \mathrm{~h}$ for zebrafish. Additional stages are added as indicated in the figure. The results obtained reveal many parallels between the different species. Thyroid hormone production starts after 71 days in man, 20 days in rat and 2.5 days $(\sim 60 \mathrm{hpf})$ in zebrafish (Bohnsack et al., 2011; Contempré et al., 1993; Strum et al., 1971). 
Figure $1 \mathrm{~b}$ provides a schematic overview of landmarks associated with the development of the thyroid and the production of T4 in zebrafish from 0 until 120 hpf. Briefly, the zebrafish thyroid gland develops from endodermal cells through a process that is in large part evolutionarily conserved (Wendl et al., 2002). The two critical transcription factors $n k x 2.1$ and pax 2.1 (functionally similar to human pax 8) are already expressed at 24 hpf (Antonica et al., 2012; Wendl et al., 2002). A fate mapping study showed that the thyroid precursor cells are located near cardiac lateral plate mesoderm (Wendl et al., 2007). Thyroglobulin gene expression starts around $32 \mathrm{hpf}$ and the expression of the sodium iodide symporter (slc5a5) at $40 \mathrm{hpf}$. A first follicle is positive for the thyroglobulin protein by $55 \mathrm{hpf}$ and for T4 by 60 hpf and expands anteriorly away from the heart, leaving a trail of thyroid follicles along the pharyngeal midline (Alt et al., 2006; Wendl et al., 2007). By 120 hpf, 6-10 thyroid follicles span along this axis from the gill region until they reach an axis that intersects the middle of the eyes. This is a time of peak T4 production, with the rise starting at around the time of hatching (72 hpf) (Chang et al., 2012). Live imaging using a transgenic zebrafish line expressing fluorescent thyroglobulin has recently provided further insight into the development of the thyroid, suggesting a role for the hypobranchial artery in guiding late thyroid expansion (Opitz et al., 2012). Figure 1c provides a schematic overview of thyroid tissue development with T4 immunohistochemistry (IHC) at $120 \mathrm{hpf}$ providing a visual reference. The first follicle to be formed (primary folli- cle) is formed near the heart during early development and expands anteriorly along the pharyngeal midline until it reaches the mid-region of the eyes, leaving behind a trail of follicular cells that later form additional follicles.

Based on this analysis, two time windows of exposure, namely $48 \mathrm{hpf}$ to $120 \mathrm{hpf}$ and 0 to $120 \mathrm{hpf}$ were chosen, with the prior avoiding the potential effect of compounds on morphogenesis of the first thyroid follicle and the second one including such effects. These thyroid-specific time windows were further compared with the exposure time window of 0-72 hpf, which was used by Hermsen et al. (2011) in order to assess the GMS of developmental toxicants. Exposures were conducted with 12 replicates per compound concentration for all time windows in one experiment. In addition, for the $0-120 \mathrm{hpf}$ time window, an additional experiment with 8 replicates was performed resulting in a total of 20 replicates per compound concentration for the 0-120 hpf time window.

\subsection{Morphological effects of thyroid-active model compounds}

Figure 2 shows an overview of the altered morphologies of the zebrafish exposed from 0 to $120 \mathrm{hpf}$ to the highest tested concentrations of the test substances. The highest tested concentration of $\mathrm{T} 3(500 \mu \mathrm{M})$ affected zebrafish embryos dramatically with an observed reduction in melanophore density and size, abnormal iridophores, deflated swim bladder, accumulation of biliverdin around the liver, and an indication of
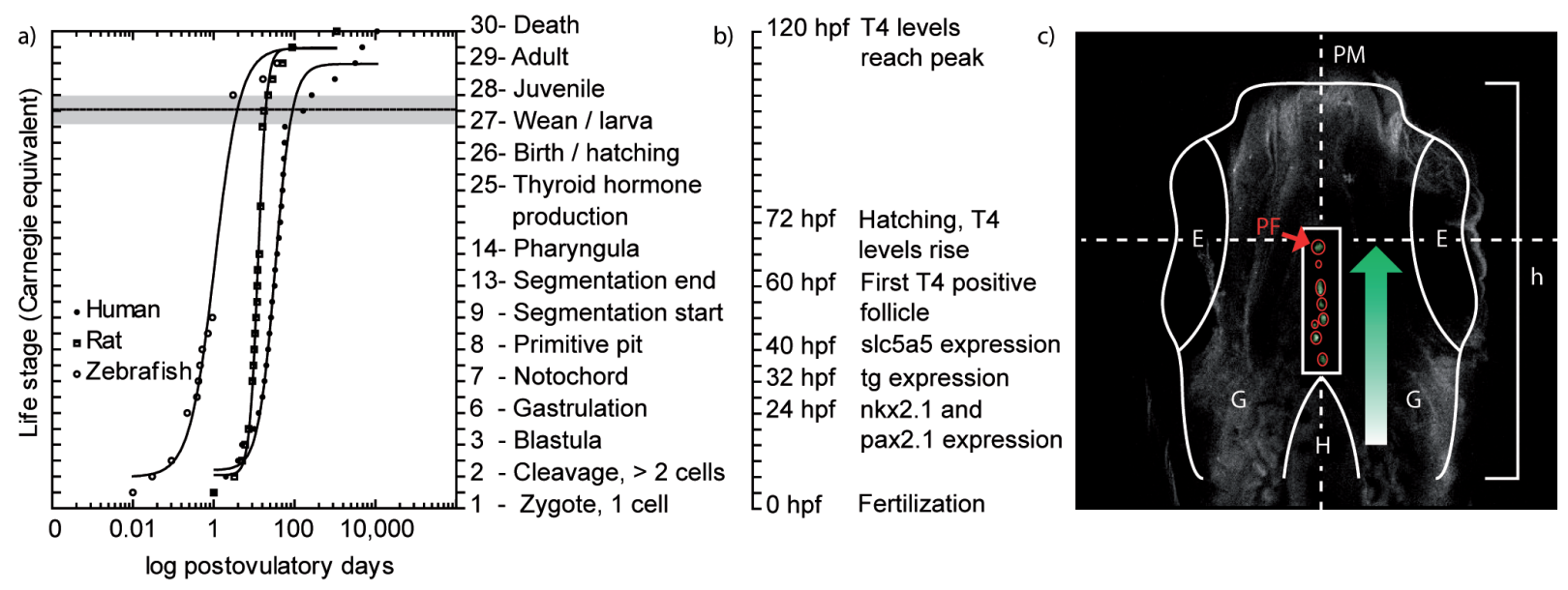

\section{Fig.1: Selection of exposure period relevant to thyroid disruption}

a) Human, rat and zebrafish developmental stages (Carnegie equivalent) plotted against time in postovulatory days. Carnegie stage 24 is the last stage of embryonic development and occurs at 33 days for humans, the equivalent of 16 days for rat and $72 \mathrm{~h}$ for zebrafish. b) Developmental landmarks of the thyroid hormone system in zebrafish from 0 to $120 \mathrm{hpf}$, including the expression of the differentiation markers nkx2.1 and pax2.1, expression of thyroglobulin (tg), the sodium iodide symporter (slc5a5), the first follicle that can be detected by $\mathrm{T} 4 \mathrm{IHC}$, as well as the rise and peak of T4 levels. c) Schematic representation of thyroid follicle development. PF stands for primary follicle (additional follicles are circled in red); $\mathrm{H}$ for heart; PM for pharyngeal midline (represented by a dotted line); $\mathrm{E}$ for eyes; $\mathrm{G}$ for gills; $\mathrm{h}$ for head region. The green arrow indicates the direction of PF development from 0 to $120 \mathrm{hpf}$. In the background, T4 IHC of a $120 \mathrm{hpf}$ zebrafish provides a visual reference. 
an adrenergic response - mainly tachycardia, which is a well documented effect of T3 - as well as dyskinesia observed as a lack of swimming behavior. In terms of teratogenicity, an observation was made for kyphosis (hunchback), however since the teratogenicity parameters used only included scoliosis (oblique bending), it was scored as such. Most effects on morphology could already be seen at $50 \mathrm{nM}$ T3. The effects were similar in the 48-120 hpf exposure window while for the 0-72 hpf time window only abnormal pigmentation, kyphosis and a mean percentage failed or delayed hatching of $41.7 \pm 1.2 \%$ (mean \pm SEM) could be observed. Intestinal convolution could be clearly observed with histological slices (Fig. 2b) but could not be clearly viewed under a stereo microscope and hence was not included as a parameter in the scoring system. Lower jaw protrusion was also clearly visible, especially with Alcian blue staining (Fig. 2c).

At doses of $250 \mu \mathrm{M}$ MMI and higher, failure to hatch was observed for the exposure periods 0-72 hpf and 0-120 hpf. For the exposure period from 48-120 hpf, only the highest tested concentration of $2 \mathrm{mM}$ led to the failed hatching of $16.7 \pm 0.9 \%$ of the zebrafish embryos, compared to $100.0 \pm 0.0 \%$ failed hatching at the highest tested concentration for 0-72 hpf and $84.2 \pm 0.4 \%$ for $0-120$ hpf. Unhatched embryos had deflated swim bladders, unresorbed yolk sacs and, as can be expected from unhatched embryos, a lack of swimming behavior.

PTU also led to failed hatching at the highest tested concentration of $400 \mu \mathrm{M}$ at a rate of $27.3 \pm 1.2 \%$ for exposures from
0-72 hpf, $11.1 \pm 0.5 \%$ from 0 - $120 \mathrm{hpf}$ while no failed hatching was observed for the exposure period from 48-120 hpf.

Upon exposure to $\mathrm{NaClO}_{4}$ from $0-120 \mathrm{hpf}$, deflated swim bladders were observed starting at $25 \mu \mathrm{M}$ up to the highest tested concentration of $400 \mu \mathrm{M}$ in an average of $12.0 \pm 0.4 \%$ of embryos, while exposure to AMI at the highest tested concentration of $10 \mu \mathrm{M}$ led to deflated swim bladders in $35.3 \pm 0.7 \%$ of embryos exposed during the same time period. No significant effects could be observed during exposures to either compound using the other time windows tested (0-72 hpf or 48-120 hpf) suggesting exposure duration to influence the sensitivity of the assay and 0-120 hpf to be the preferred time window for exposure.

\subsection{Adjusted morphological scoring: general development score}

The GMS scoring system was developed to look at the effect of chemicals on zebrafish development at $72 \mathrm{hpf}$ (Hermsen et al., 2011). Based on the results obtained here with the selected thyroid modulating compounds, four main adjustments were made to the GMS scoring system. First, 120 hpf-specific developmental endpoints affected by thyroid-active compounds were added to the GMS. These endpoints included reflective iridophores (Fig. 3), beat and glide swimming, inflation of swim bladder and resorption of yolk sac (Fig. 2). Since these endpoints are not included in the GMS for 0-72 hpf, they were incorporated in a new scoring system denoted general devel-

a)

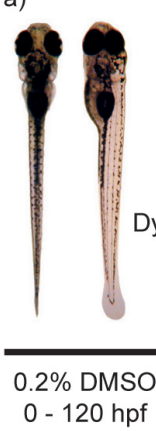

b)

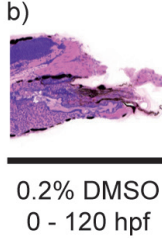

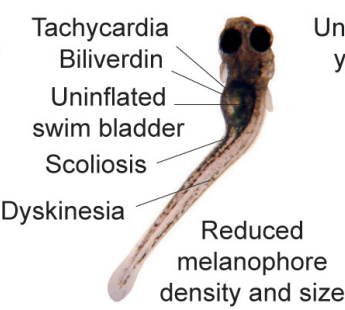

density and size Unhatched

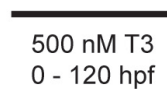

Intestinal convolution

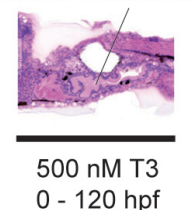

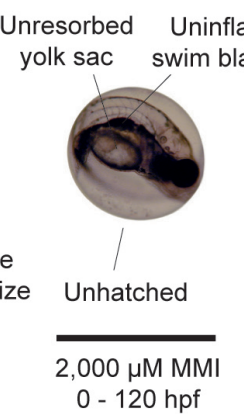

c)

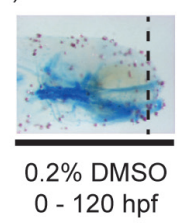

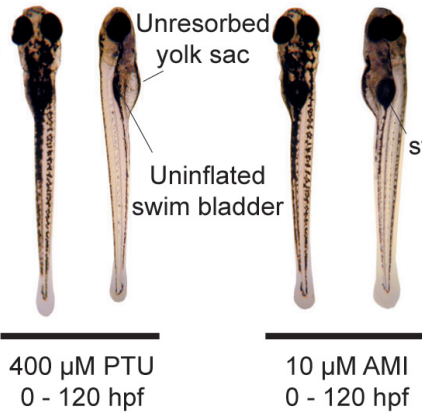

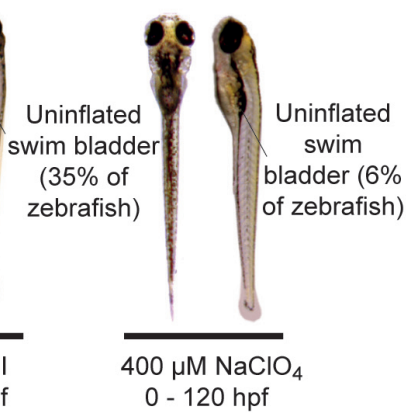

Lower jaw protrusion

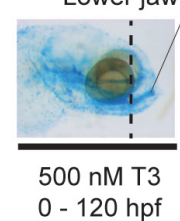

Fig. 2: Changes in morphology following exposure to thyroid-active compounds

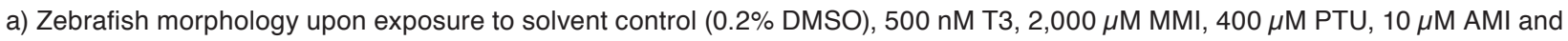
$400 \mu \mathrm{M} \mathrm{ClO}_{4}$. b) Sagittal section of zebrafish exposed to $500 \mathrm{nM}$ T3 stained with hematoxylin and eosin (H\&E) reveals a high degree of intestinal convolution that is absent in the solvent control. c) Alcian blue staining of the cartilage revealing the anterior-dorsal protrusion of the lower jaw following exposure to 500 nM T3 from 0-120 hpf. 


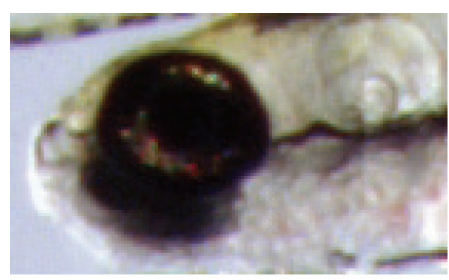

$0.2 \%$ DMSO

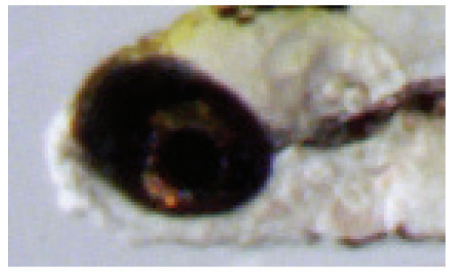

$0.05 \mathrm{nM} \mathrm{T3}$

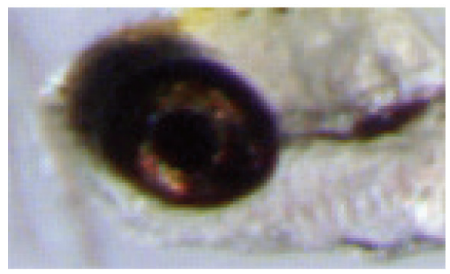

$0.5 \mathrm{nM}$ T3

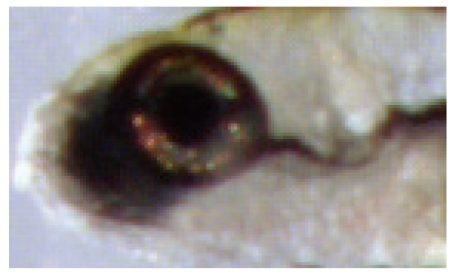

$5 \mathrm{nM}$ T3

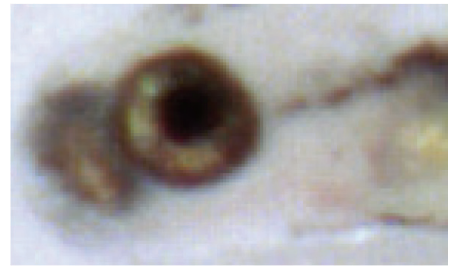

$50 \mathrm{nM}$ T3

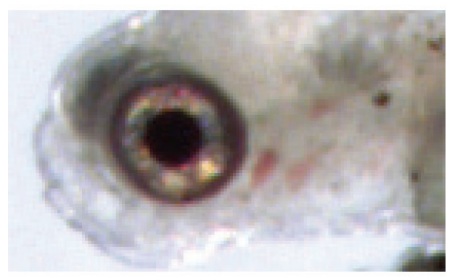

$500 \mathrm{nM} \mathrm{T3}$

Fig. 3: Dose-dependent increase in zebrafish iridophore pigmentation following exposure to $\mathrm{T} 3$

Lateral view of $120 \mathrm{hpf}$ zebrafish showing iridophore pigmentation (reflective pigments) increasing dose-dependently upon exposure to T3. opment score (GDS). Secondly, a refinement was made in the newly defined GDS by combining both morphology and teratogenicity endpoints in a similar way to the total morphological score (TMS) applied in the method of Piersma et al. (2004) for the rat whole embryo culture (WEC) which combines teratogenicity and developmental toxicity (Fig.4). Thirdly, a refined scoring approach was also implemented similar to that used by Teixidó et al. (2013) whereby developmental abnormalities for an endpoint are treated as a lack of development and therefore result in a score of 0 for that developmental endpoint compared to a score of 1 per event for normal development. Fourthly, the terms used for developmental abnormalities are compliant with major databases with the use of the Entity Quality Ontology (EQO) convention suggested by the Open Biomedical Ontologies (OBO) Consortium, which involves describing an entity (e.g. tail vasculature) and a quality (e.g., edema) (Balhoff et al., 2011; Dahdul et al., 2010). This leaves room for the standardized annotation of additional abnormalities based on anatomical location or feature (such as movement). Database compliance also enables computable phenotypes as well as the development of iterative models that improve the accuracy of future chemoinformatic predictions. Examples of commonly observed abnormalities are included in Figure 4 for reference purposes. Minor adjustments to the highlighted abnormalities include the addition of visible biliverdin or bilirubin as well as the changing of the term scoliosis to spinal curvature in order to encompass all forms of a bent spine, which include kyphosis and lordosis in addition to scoliosis (Fig. 4).

\subsection{GMS compared to GDS}

The GMS for the highest concentration of T3 $(500 \mu \mathrm{M})$ decreased to $12.9 \pm 0.7(n=12)$ for the exposure period from $0-72 \mathrm{hpf}, 11.3 \pm 0.5(\mathrm{n}=12)$ from $48-120 \mathrm{hpf}$ and $11.1 \pm 2.6$ from $0-120 \mathrm{hpf}(\mathrm{n}=20)$, as compared to a value of $15.0 \pm 0.0$ for the unexposed control. The GDS for the highest concentration of T3 was $12.2 \pm 3.7(n=20)$ from $0-120 \mathrm{hpf}$ as compared to18.8 \pm 0.7 for the control. For better comparison, normal growth was set as a common denominator with a baseline value of 1 , and the fold change relative to the controls was also calculated. The fold change relative to the controls for the two highest concentrations of T3 (1-120 hpf, $\mathrm{n}=20)$ went from 0.9 and 0.7 in the GMS to 0.8 and 0.6 in the GDS, respectively, thereby increasing the statistical significance from $\mathrm{p} \leq 0.01$ to $\mathrm{p} \leq 0.0001$.

The GMS at the highest tested concentration of MMI $(2,000$ $\mu \mathrm{M}$ ) was $13.1 \pm 0.3$ as an average of all exposure periods, which represents a fold change of 0.9 relative to the control (GMS = $15.0 \pm 0.3)$. The GDS at the highest tested concentration of MMI was $12.8 \pm 2.7(0-120 \mathrm{hpf})$, which represents a fold change of 0.7 relative to the control (GDS $=18.4 \pm 1.6$; $\mathrm{p} \leq 0.0001)$. Moreover, statistical significance could be reached at the lower concentration of $1,000 \mu \mathrm{M}(\mathrm{p} \leq 0.05)$ with the GDS but not the GMS.

Figure 5 gives an overview of the effects of T3, MMI, PTU, and $\mathrm{ClO}_{4}$ on zebrafish development, in both GMS and GDS, represented as fold change relative to the controls. These tested compounds led to developmental abnormalities at the highest tested concentration and the GDS was better able to reflect these abnormalities than the GMS. 


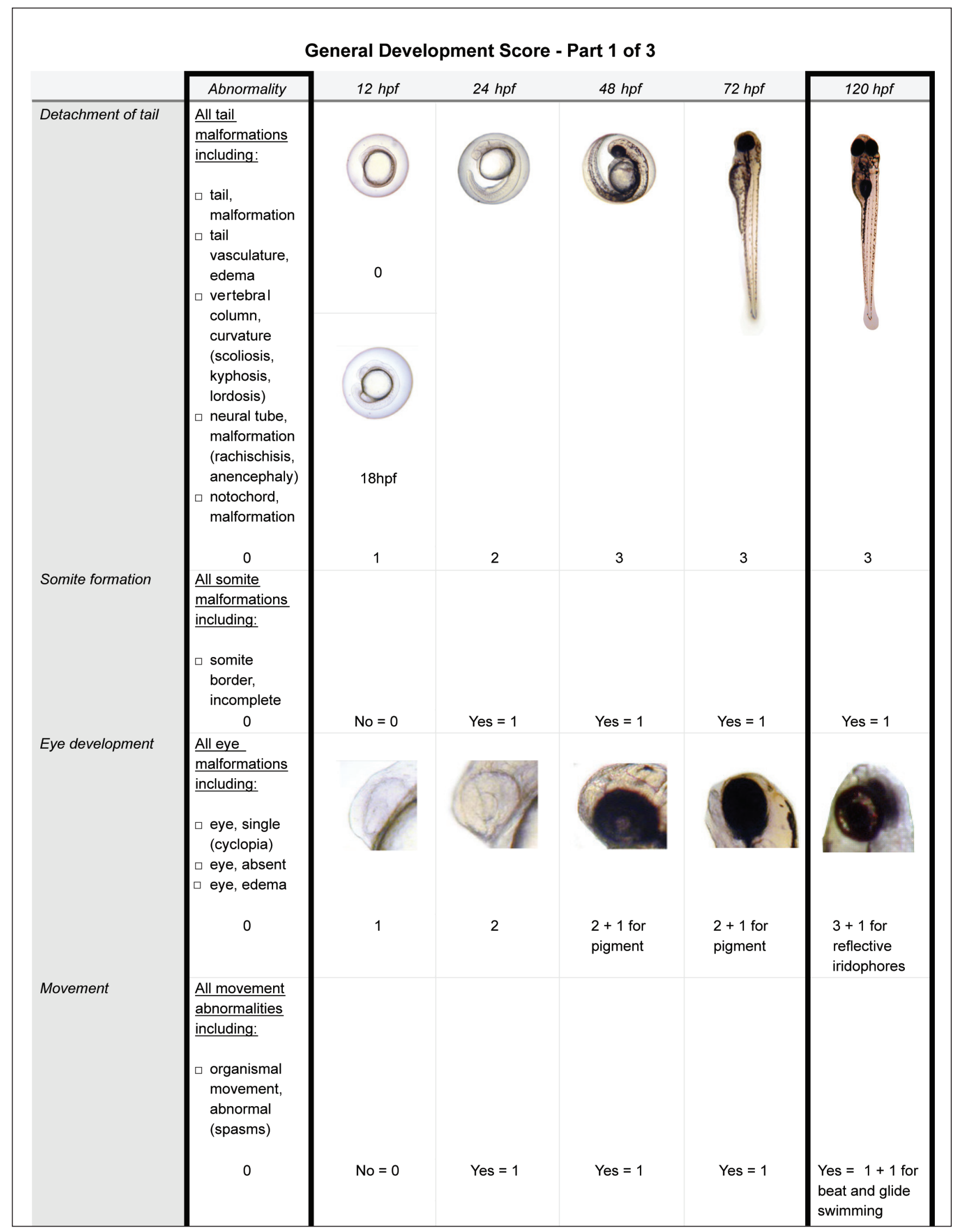




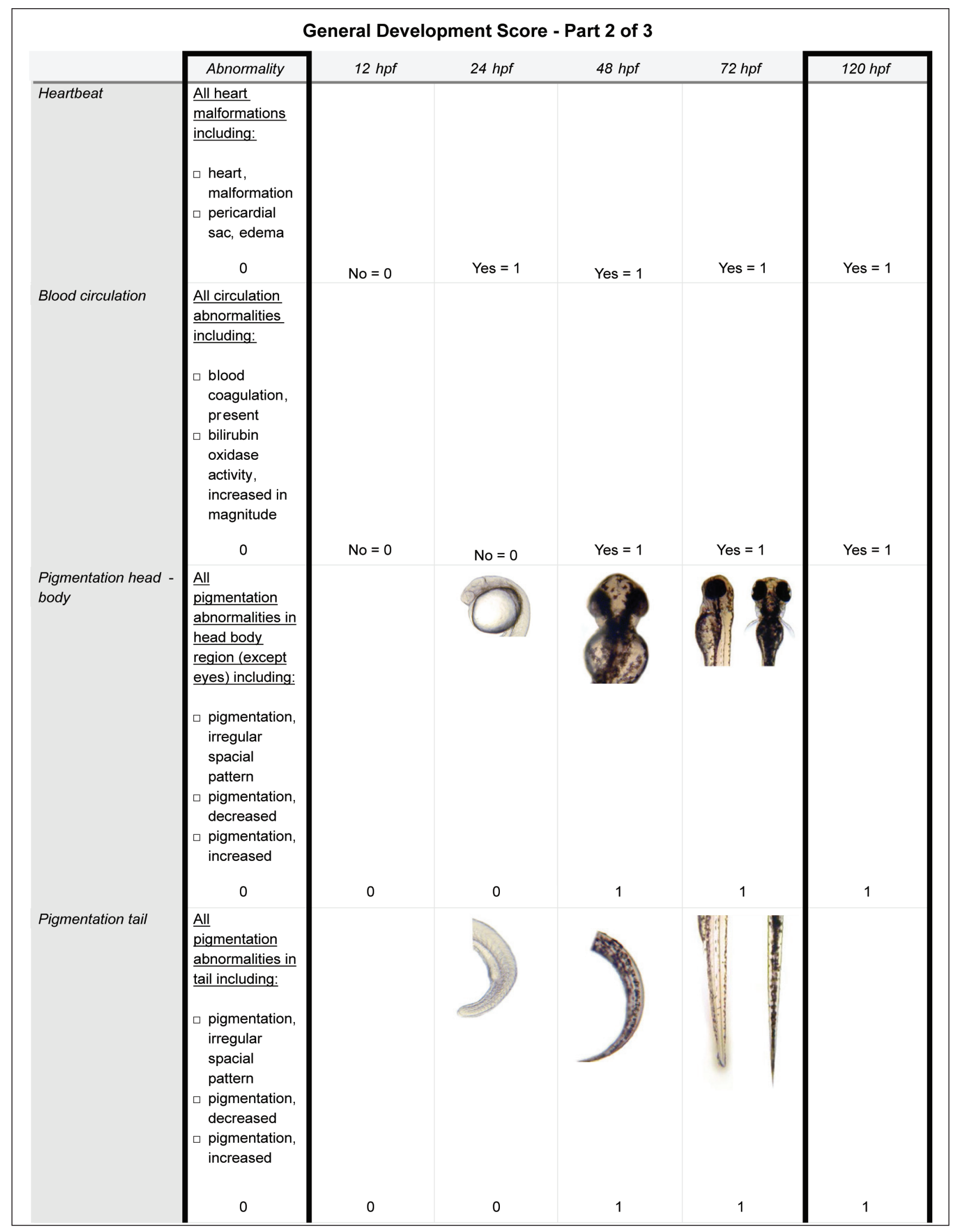




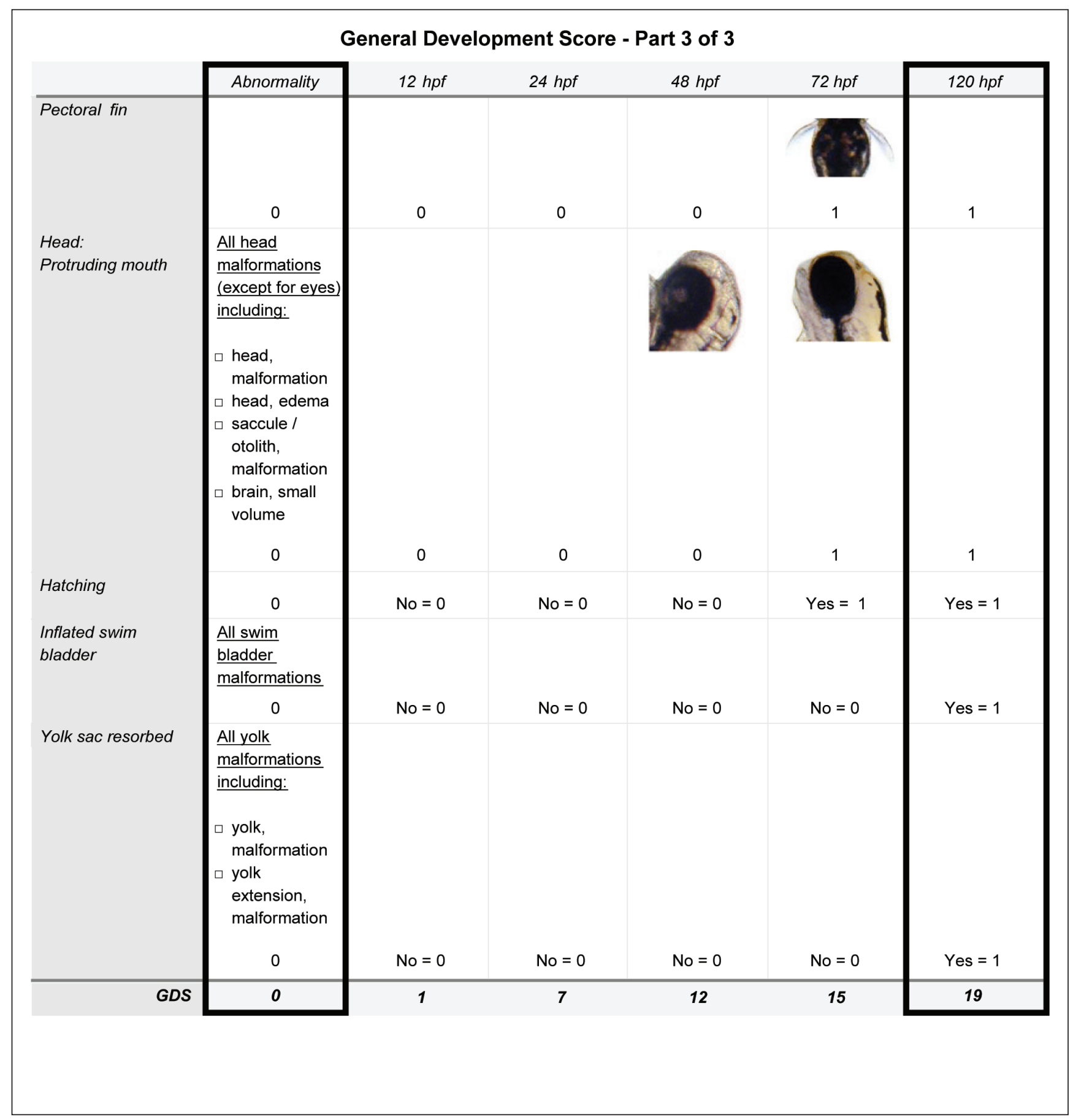

Fig. 4: The GDS system for the scoring of general development

GDS system for the assessment of general developmental toxicity at $120 \mathrm{hpf}$. The system takes into account morphology, dysmorphology as well as non-morphological parameters such as swimming. Additions made to the GMS scoring system (Hermsen et al., 2011) in order to better accommodate effects of thyroid-active compounds are highlighted with a bold outline. 

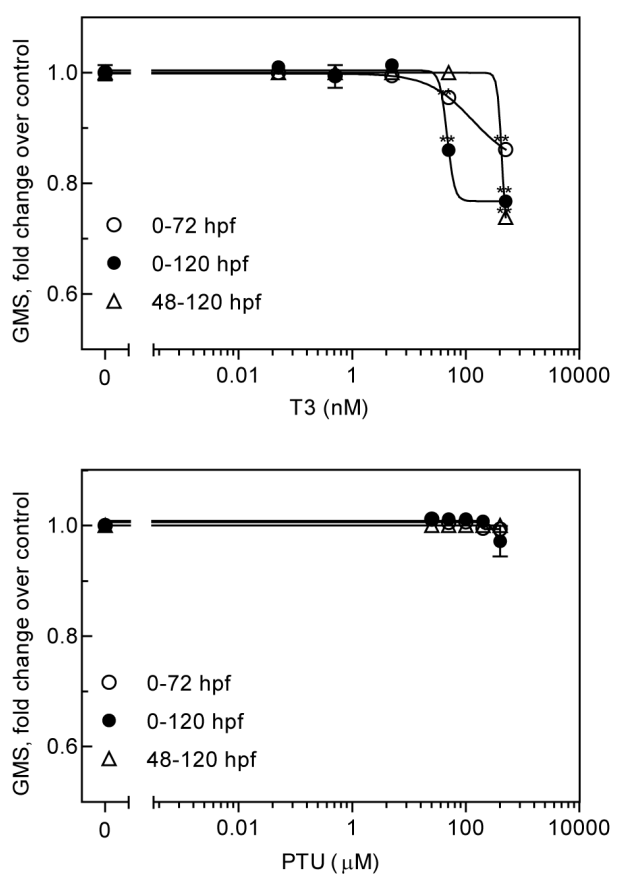

e)

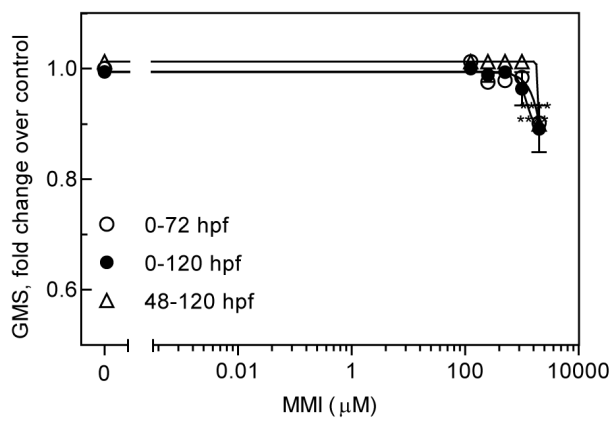

g)

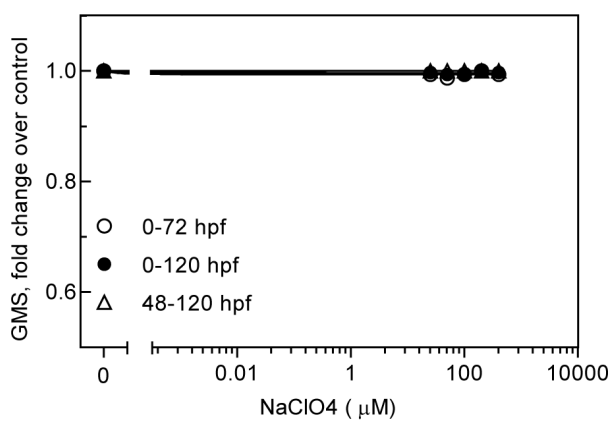

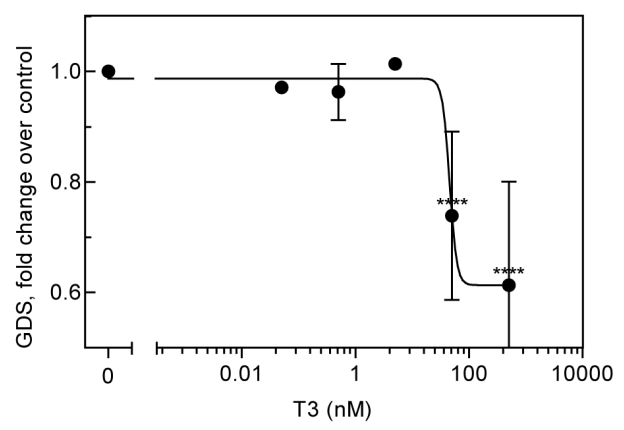

d)
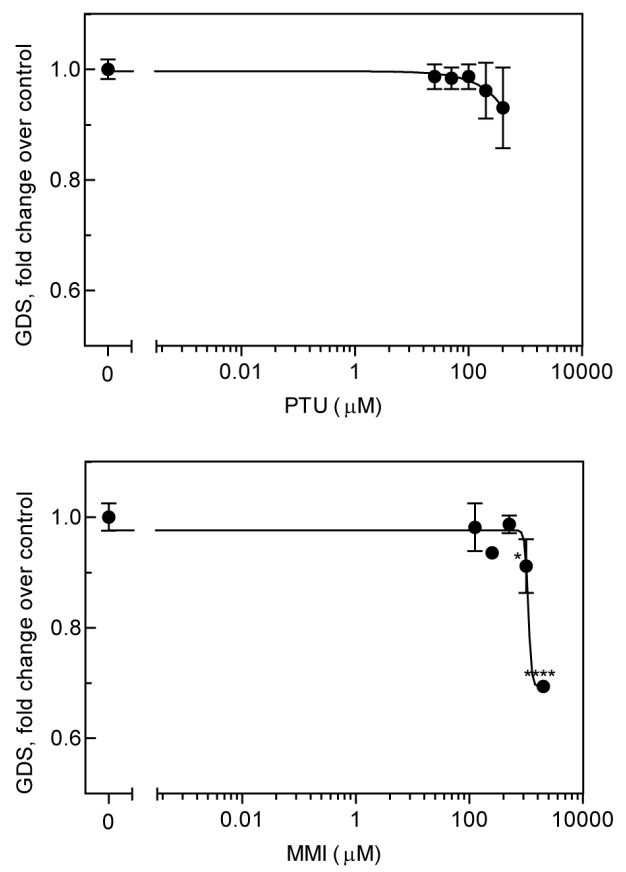

h)

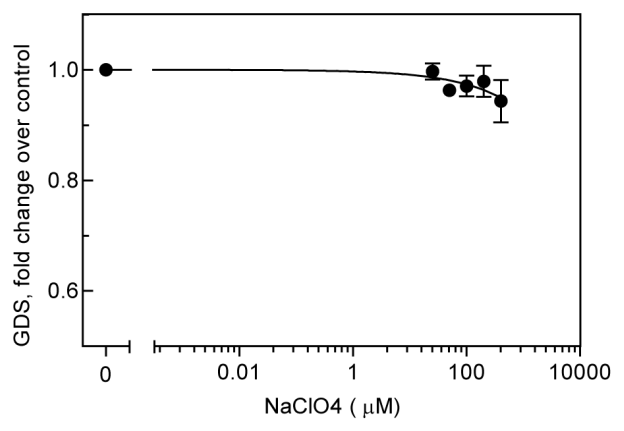

Fig. 5: T3, PTU, MMI and $\mathrm{NaClO}_{4}$-induced alterations in development assessed by both the GMS and GDS

Effect of T3 exposure on a) GMS and b) GDS; PTU exposure on c) GMS and d) GDS; MMI exposure on e) GMS and f) GDS; CIO 4 exposure on g) GMS and h) GDS. Results are based on 12 replicates per concentration $(n=12)$ and since the total scores are different, these are expressed as fold decrease relative to the full score (normal development). A top down approach was used to minimize the number of embryos used and the $0-120 \mathrm{hpf}$ exposure window, which was found to be the most appropriate time window for the thyroidrelated parameters studied, was selected for an additional independent experiment at a separate laboratory with 8 replicates per concentration ( $n=8$; a total of $n=20$ for $0-120$ hpf experiments). The error bars are shown for only this time window as the standard error of the mean of these two experiments $(\mathrm{N}=2)$. The calculation of statistical significance was done by pooling all replicates from each experiment for each concentration and comparing to the $0.2 \%$ DMSO solvent controls in a two-tailed student's t-test.

${ }^{*} \mathrm{P} \leq 0.05$; ${ }^{* *} \mathrm{P} \leq 0.01$; ${ }^{* * *} \mathrm{P} \leq 0.001 ;{ }^{* * *} \mathrm{P} \leq 0.0001$. 

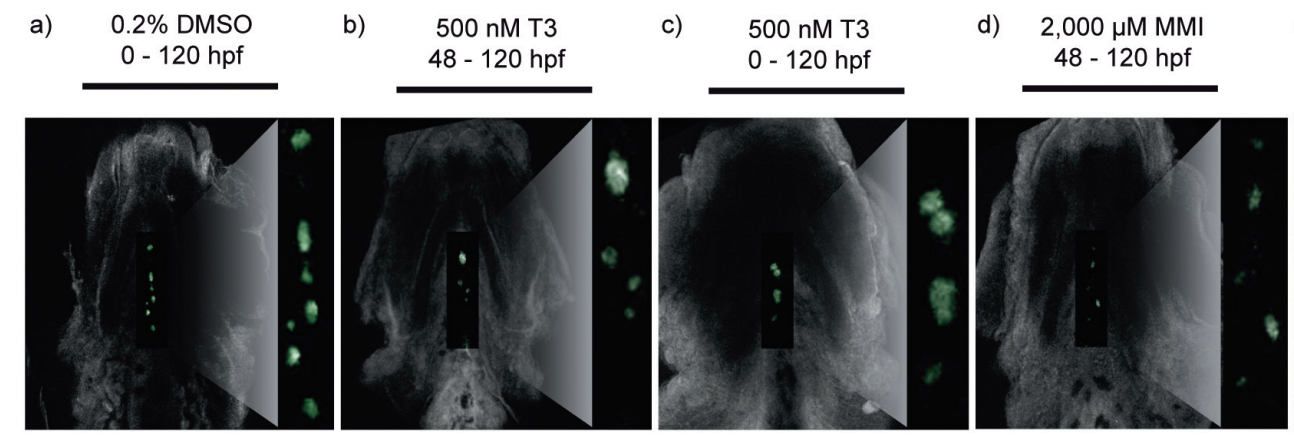

e) 2,000 $\mu \mathrm{M}$ MMI $0-120 \mathrm{hpf}$

Relative fluorescence intensity (RFU): Number of follicles:

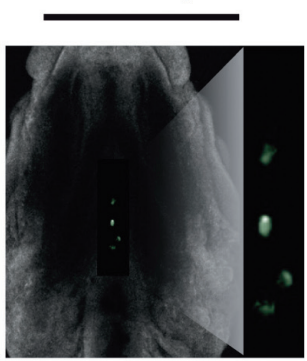

Fig. 6: Effect of T3 and MMI on T4 immunofluorescence in zebrafish thyroid follicles

T4 whole mount immunohistochemistry upon exposure of zebrafish to a) $0.2 \%$ DMSO as the solvent control from 0-120 hpf; $500 \mathrm{nM}$ T3 from b) 48-120 hpf and c) 0-120 hpf; and 2,000 $\mu \mathrm{M} \mathrm{MMI} \mathrm{from} \mathrm{d)} \mathrm{48-120} \mathrm{hpf} \mathrm{and} \mathrm{e)} \mathrm{0-120} \mathrm{hpf.} \mathrm{Thyroid} \mathrm{follicles} \mathrm{were} \mathrm{tested} \mathrm{for} \mathrm{the} \mathrm{signal}$ intensity of T4 immunofluorescence and expressed as relative fluorescence units (RFU). The number of follicles was counted based on the images taken with the confocal laser scanning microscope (the region of interest is enlarged and presented at the right side of each panel). T3 exposure at the highest tested concentration and both time points resulted in a decrease in the number of follicles. $\mathrm{MMI}$ exposure at the highest concentration resulted in both lower T4 signal intensity and lower number of follicles.

A comparison of the exposure time windows of 0-72 hpf and 0-120 hpf, reveals that the most significant results were achieved with exposures from 0-120 hpf (Fig. 5). The time window of 48-120 hpf did not add any additional information or significance compared to the other time windows. In the GDS, T3 and MMI exposure resulted in a no observed adverse effect level (NOAEL) of $5 \mathrm{nM}$ and $500 \mu \mathrm{M}$, respectively. The GDS for PTU, amiodarone and $\mathrm{ClO}_{4}$ did not change significantly and therefore a NOAEL could not be established for these compounds.

\subsection{T4 immunohistochemistry}

The model compounds tested were selected based on their known ability to alter thyroid homeostasis and only those that significantly affected the GDS score, namely T3 and MMI, were further tested for thyroid status by T4 immunohistochemistry (IHC) in order to confirm that the developmental toxicity detected by the GDS for these compounds was accompanied by an effect on the thyroid system (Fig. 6). Under control conditions, the thyroid follicles are well spaced out anteriorly by $120 \mathrm{hpf}$. In zebrafish exposed to T3 both number of follicles and their spread were affected compared to the control (Fig. 6a), indicating potential thyroid dysmorphogenesis, while MMI exposure led to a clear drop in signal intensity and in the number of follicles compared to the control (Fig. 6b,c), confirming a drop in follicular T4 levels.

\section{Discussion}

Critical to the proper development of the vertebrate embryo, thyroid homeostasis is prone to disruption by man-made chemi- cals with an ability to act through very diverse modes of action and within multiple organs (Hartoft-Nielsen et al., 2011; Jomaa, 2014; Tan et al., 2007). Given that alterations in the thyroid system can have severe effects on development (Boyages and Halpern, 1993; Haddow et al., 1999; Utiger, 1999), the aim of the present study was to investigate whether a zebrafish-based developmental toxicity assay would be able to detect thyroid hormone-active compounds.

The pathways involved in human thyroid development are conserved in the zebrafish and so are its tissue architecture, function and feedback regulation by the HPT axis, making it a promising model for the study of thyroid-active compounds (Bourque and Houvras, 2011; Jomaa et al., 2013; Raldúa and Babin, 2009).

T3, acting through its nuclear receptor, is a morphogenic agent in certain animal species, controlling the metamorphosis of tadpoles and frogs (Shi et al., 1996). In the present study, T3 exposed zebrafish clearly showed significant developmental toxicity evident as scoliosis, abnormal iridophores, reduced melanophore density and size as well as a lack of an inflated swim bladder (Fig. 4). Moreover, T3 exposed zebrafish showed signs of acute toxicity such as excess biliverdin that is visible under a stereo microscope, tachycardia and dyskinesia. The latter two are symptomatic of an adrenergic response and are likely a result of T3 increasing beta-adrenergic receptor density (Mooradian and Scarpace, 1993). A high rate of $42 \%$ failed hatching occurred only at the 0-72 hpf exposure window. Increased sensitivity for hatching failure is expected at $72 \mathrm{hpf}$ since that is around the time hatching occurs. However, as can be seen in Figure 5, overall sensitivity to thyroid-active com- 
pounds is higher in the GDS at $0-120 \mathrm{hpf}$ as it includes additional parameters that are specific to that time window.

PTU and MMI have a direct effect on thyroglobulin iodination by thyroid peroxidase, thereby inhibiting thyroid hormone synthesis (Taurog, 1976). These compounds are used medically to treat hyperthyroidism. A decrease in the circulating T3 levels can affect various developmental endpoints including intestines, brain and reproductive capacity. Raldúa and Babin (2009) reported a complete lack of T4 immunoreactivity beyond 0.75 $\mathrm{mM}$ and $0.18 \mathrm{mM}$ in zebrafish for MMI and PTU, respectively. This reduction in thyroid hormone levels was accompanied by a reduction in the GDS (Fig. 5). Moreover, the hatching rate was strikingly low in the MMI-treated group and this was deemed significant, not only statistically, but also physiologically. This result is in line with the observation that chicken embryos have been reported to have delayed hatching upon exposure to MMI (Haba et al., 2011). Interestingly, another compound shown to affect zebrafish hatching rate is perfluorooctanoic acid (PFOA), an established thyroid-active compound (Lopez-Espinosa et al., 2012; Melzer et al., 2010; Zheng et al., 2011). Hence, the change in zebrafish hatching rate that was induced by thyroid active compounds was accurately reflected in the GDS developmental toxicity assay (Fig. 5). Moreover, MMI exposure resulted in a NOAEL of $500 \mu \mathrm{M}$ in the GDS, which is several orders of magnitude higher than the T3 NOAEL of $5 \mathrm{nM}$, and this relative potency compares well with the NOAEL based on changes in thyroid stimulating hormone (TSH) levels derived from rodent studies (a NOAEL of $0.5 \mathrm{mg} / \mathrm{kg} \mathrm{bw} /$ day for MMI and a NOAEL of less than $10 \mu \mathrm{g} / \mathrm{kg}$ bw/day for T3) (Grover et al., 2007; Hood et al., 1999).

$\mathrm{NaClO}_{4}$ inhibits sodium iodide symporter (NIS) transport of iodide into the thyroid follicular cells, thereby resulting in a decline in thyroid hormones and a rise in thyrotropin (Stoker et al., 2006). The morphological changes observed in the developing zebrafish embryo included uninflated swim bladder and incomplete resorption of the yolk sac. While these were consistent observations that could only be made by including $120 \mathrm{hpf}$-specific parameters, they did not have enough weight within either the GMS or GDS system to significantly change the overall score. This corroborates in vivo data on altered thyroid hormone levels in rodent studies showing that $\mathrm{NaClO}_{4}$ is less potent than either PTU or MMI (Hood et al., 1999; Stoker et al., 2006; Yamasaki et al., 2002). Moreover, a human epidemiological study failed to find an association between perchlorate levels in drinking water and congenital defects of the thyroid (Crump et al., 2000). In another study with volunteers given perchlorate in drinking water the administered dose was negatively correlated with thyroidal iodide uptake but even a $70 \%$ decrease in iodide transport did not result in altered T4 levels (Greer et al., 2002; Strawson et al., 2004). The relatively low developmental toxicity of perchlorate is supported by animal studies showing that pregnant rats and rabbits exposed to a perchlorate dose as high as $30 \mathrm{mg} / \mathrm{kg} \mathrm{bw} /$ day do not give birth to offspring with signs of developmental toxicity and changes in thyroid hormone levels were seen as adaptive and reversible (York et al., 2003, 2005).

Amiodarone is an anti-arrhythmic agent (heart), beta blocker and calcium blocker. Its chemical structure resembles that of $\mathrm{T} 4$ and the compound alters thyroid hormone levels in vivo (de Sandro et al., 1991), likely due to its high iodide content (Pitsiavas, 1999). In zebrafish, amiodarone was reported to lead to a significant reduction in $\mathrm{T} 4$ immunoreactivity at $1 \mu \mathrm{M}$ (Raldúa and Babin, 2009). However, while morphological changes were observed in the present study upon exposure to amiodarone, such as the lack of an inflated swim bladder in some animals, which is included in the GDS, statistical significance could not be reached in both the GMS and GDS (data not shown). Indeed, human studies on amiodarone treated mothers indicate that transient hypothyroidism in the newborn may occur but there is no adverse effect on development (Matsumura et al., 1992; Valensise et al., 1992).

The thyroid-active compound associated phenotypes observed in 120 hpf zebrafish include 1) hatching (discussed in the previous section), 2) yolk sac resorption, 3) iridiophore pigmentation, 4) melanophore pigmentation, 5) swim bladder inflation, 6) bent spines, 7) beat and glide swimming, 8) lower jaw cartilage protrusion and 9) maturation of the gastrointestinal system, with the last two being better suited for staining techniques or high resolution microscopy for accurate assessment. These developments occur concurrently with a surge in thyroid hormone levels, which reach a peak at $120 \mathrm{hpf}$ (Chang et al., 2012). The overall results have indicated that the time window of $0-120 \mathrm{hpf}$ is better able to reflect the effect of thyroid-active compounds on zebrafish development than the other time windows studied (Fig. 5) and support the suggestion by Liu and Chan that there is a distinct thyroid hormone-mediated embryonic to larval transition in zebrafish that is analogous to the thyroid hormone-mediated larval to juvenile transition originally proposed by Brown (Brown, 1997; Liu and Chan, 2002).

The period from the time the zebrafish embryo has hatched (72 hpf) until the time it is free swimming (120 hpf) is termed the yolk-sac larva (Balon, 1990). The resorption of the yolk sac and hence the maternal food supply is only evident at around $120 \mathrm{hpf}$, with total resorption evident by around $168 \mathrm{hpf}$ (Jardine and Litvak, 2003; Kimmel et al., 1995). The role of thyroid hormones in this developmental process has been previously documented whereby MMI co-administered with AMI resulted in the retardation of yolk sac resorption and this was rescued with T4 (Liu and Chan, 2002).

Overall, the results obtained in this paper show the importance of the 0-120 hpf time window of zebrafish exposure in the assessment of the developmental toxicity of thyroid-active compounds. The newly developed GDS scoring system takes into account parameters that occur after $72 \mathrm{hpf}$. The defined GDS can successfully detect zebrafish developmental toxicity induced by thyroid-active compounds and can benefit from the model organism's potential for high-throughput, which, for screening purposes, is a key advantage over rodent assays. The parameters affected, which include reflective iridophores, beat and glide swimming, inflated swim bladders, and resorbed yolk sacs, are shown to be sensitive towards toxicity induced by $\mathrm{TH}$ disrupting compounds. One could envisage that other compounds causing developmental toxicity by different modes of 
action may also affect these parameters. Since this opens the way to false positives if the assay were to be used in isolation to detect TH activity, we envision that the GDS could be part of an ITS that includes specific biomarkers of thyroid hormone disruptor activity. Although the GDS will detect developmental toxicity resulting from various modes of action, it is shown in the present study that the GDS can be used to flag compounds as potential thyroid disruptors. Due to the presence of an intact thyroid hormone feedback loop, the zebrafish represents an experimental in vitro platform that is vastly more comprehensive than individual cell lines. Moreover, the zebrafish embryo up to $120 \mathrm{hpf}$ is more socially-acceptable as a test organism than higher vertebrates. When applied in combination with other in vitro tests within an ITS, the zebrafish GDS holds promise to reduce, refine and, in certain cases, replace animal testing.

\section{References}

Alt, B., Reibe, S., Feitosa, N. M. et al. (2006). Analysis of origin and growth of the thyroid gland in zebrafish. Dev Dynam 235, 1872-1883. http://dx.doi.org/10.1002/dvdy.20831

Antonica, F., Kasprzyk, D. F., Opitz, R. et al. (2012). Generation of functional thyroid from embryonic stem cells. Nature 491, 66-71. http://dx.doi.org/10.1038/nature11525

Balhoff, J. P., Dahdul, W. M., Lapp, H. et al. (2011). Employing reasoning within the phenoscape knowledgebase. In O. Bodenreider, M. E. Martone and A. Ruttenberg (eds.), ICBO, CEUR Workshop Proceedings, CEUR-WS.org.

Balon, E. K. (1990). Epigenesis of an epigeneticist: The development of some alternative concepts on the early ontogeny and evolution of fishes. Guelph Ichthyol Rev, 1-48.

Bohnsack, B. L., Gallina, D. and Kahana, A. (2011). Phenothiourea sensitizes zebrafish cranial neural crest and extraocular muscle development to changes in retinoic acid and IGF signaling. PLOS ONE 6, e22991. http://dx.doi. org/10.1371/journal.pone.0022991

Bourque, C. and Houvras, Y. (2011). Hooked on zebrafish: Insights into development and cancer of endocrine tissues. Endocr-Relat Cancer 18, R149-R164. http://dx.doi. org/10.1530/ERC-11-0099

Boyages, S. C. and Halpern, J. P. (1993). Endemic cretinism: toward a unifying hypothesis. Thyroid 3, 59-69. http:// dx.doi.org/10.1089/thy.1993.3.59

Brown, D. D. (1997). The role of thyroid hormone in zebrafish and axolotl development. PNAS 94, 13011-13016. http:// dx.doi.org/10.1073/pnas.94.24.13011

Chang, J., Wang, M., Gui, W. et al. (2012). Changes in thyroid hormone levels during zebrafish development. Zool Sci 29, 181-184. http://dx.doi.org/10.2108/zsj.29.181

Contempré, B., Jauniaux, E., Calvo, R. et al. (1993). Detection of thyroid hormones in human embryonic cavities during the first trimester of pregnancy. JCEM 77, 1719-1722.

Crump, C., Michaud, P., Téllez, R. et al. (2000). Does perchlorate in drinking water affect thyroid function in newborns or school-age children? J Occup Environ Med 42, 603-612. http://dx.doi.org/10.1097/00043764-200006000-00009
Dahdul, W. M., Lundberg, J. G., Midford, P. E. et al. (2010). The teleost anatomy ontology: Anatomical representation for the genomics age. Syst Biol 59, 369-383. http://dx.doi. org/10.1093/sysbio/syq013

de Sandro, V., Chevrier, M., Boddaert, A. et al. (1991). Comparison of the effects of propylthiouracil, amiodarone, diphenylhydantoin, phenobarbital, and 3-methylcholanthrene on hepatic and renal T4 metabolism and thyroid gland function in rats. Toxicol Appl Pharm 111, 263-278. http://dx.doi.org/10.1016/0041-008X(91)90030-I

ECHA (2009). New study inaccurate on the number of test animals for REACH. http://echa.europa.eu/ documents/10162/17096/pr_09_11_animal_testing 20090828_en.pdf

European Council (1986). Council Directive 86/609/EEC of 24 November 1986 on the approximation of laws, regulations and administrative provisions of the Member States regarding the protection of animals used for experimental and other scientific purposes. Off J Europ Union L 358, 1-28.

Greer, M. A., Goodman, G., Pleus, R. C. et al. (2002). Health effects perchlorate contamination: The dose response for inhibition of thyroidal radioiodine uptake in humans. Environ Health Persp 110, 927-937. http://dx.doi.org/10.1289/ ehp.02110927

Grover, G. J., Dunn, C., Nguyen, N.-H. et al. (2007). Pharmacological profile of the thyroid hormone receptor antagonist NH3 in rats. J Pharmacol Exp Ther 322, 385-390. http:// dx.doi.org/10.1124/jpet.106.116152

Haba, G., Nishigori, H., Tezuka, Y. et al. (2011). Effect of antithyroid drug on chick embryos during the last week of development: Delayed hatching and decreased cerebellar acetylcholinesterase activity. J Obstet Gynaecol Res 37, 1549-1556. http://dx.doi.org/10.1111/j.14470756.2011.01573.x

Haddow, J. E., Palomaki, G. E., Allan, W. C. et al. (1999). Maternal thyroid deficiency during pregnancy and subsequent neuropsychological development of the child. $N$ Engl J Med 341, 549-555. http://dx.doi.org/10.1056/ NEJM199908193410801

Hartoft-Nielsen, M.-L., Boas, M., Bliddal, S. et al. (2011). Do thyroid disrupting chemicals influence foetal development during pregnancy? J Thyroid Res 2011, 1-14. http://dx.doi. org/10.4061/2011/342189

Hartung, T. and Rovida, C. (2009). Chemical regulators have overreached. Nature 460, 1080-1081. http://dx.doi. org/10.1038/4601080a

Hermsen, S. A. B., van den Brandhof, E.-J., van der Ven, L. T. M. et al. (2011). Relative embryotoxicity of two classes of chemicals in a modified zebrafish embryotoxicity test and comparison with their in vivo potencies. Toxicol In Vitro 25, 745-753. http://dx.doi.org/10.1016/j.tiv.2011.01.005

Hood, A., Liu, Y. P., Gattone, V. H., 2nd et al. (1999). Sensitivity of thyroid gland growth to thyroid stimulating hormone (TSH) in rats treated with antithyroid drugs. Toxicol Sci 49, 263-271. http://dx.doi.org/10.1093/toxsci/49.2.263 
Jardine, D. and Litvak, M. K. (2003). Direct yolk sac volume manipulation of zebrafish embryos and the relationship between offspring size and yolk sac volume. $J$ Fish Biol 63, 388-397. http://dx.doi.org/10.1046/j.10958649.2003.00161.x

Jomaa, B., Aarts, J. M. M. J. G., Haan, L. H. J. de et al. (2013). In vitro pituitary and thyroid cell proliferation assays and their relevance as alternatives to animal testing. ALTEX 30, 293-307. http://dx.doi.org/10.14573/altex.2013.3.293

Jomaa, B. (2014). Thyroid toxicogenomics: A multi-organ paradigm. In J. Kleinjans (ed.), Toxicogenomics-Based Cellular Models: Alternatives to Animal Testing for Safety Assessment (159-180). Elsevier Academic Press. http://dx.doi. org/10.1016/B978-0-12-397862-2.00009-7

Kimmel, C. B., Ballard, W. W., Kimmel, S. R. et al. (1995). Stages of embryonic development of the zebrafish. Dev Dynam 203, 253-310. http://dx.doi.org/10.1002/ aja.1002030302

Liu, Y.-W. and Chan, W.-K. (2002). Thyroid hormones are important for embryonic to larval transitory phase in zebrafish. Differentiation 70, 36-45. http://dx.doi.org/10.1046/j.14320436.2002.700104.X

Lopez-Espinosa, M.-J., Mondal, D., Armstrong, B. et al. (2012). Thyroid function and perfluoroalkyl acids in children living near a chemical plant. Environ Health Perspect 120, 1036-1041. http://dx.doi.org/10.1289/ehp.1104370

Matsumura, L. K., Born, D., Kunii, I. S. et al. (1992). Outcome of thyroid function in newborns from mothers treated with amiodarone. Thyroid 2, 279-281. http://dx.doi.org/10.1089/ thy.1992.2.279

Melzer, D., Rice, N., Depledge, M. H. et al. (2010). Association between serum perfluorooctanoic acid (PFOA) and thyroid disease in the U.S. national health and nutrition examination survey. Environ Health Perspect 118, 686-692. http://dx.doi.org/10.1289/ehp.0901584

Mooradian, A. D. and Scarpace, P. J. (1993). 3,5,3'-L-triiodothyronine regulation of beta-adrenergic receptor density and adenylyl cyclase activity in synaptosomal membranes of aged rats. Neurosci Lett 161, 101-104. http://dx.doi. org/10.1016/0304-3940(93)90150-J

Nagel, R. (2002). DarT: The embryo test with the Zebrafish Danio rerio - a general model in ecotoxicology and toxicology. ALTEX 19, Suppl 1, 38-48. http://www.altex.ch/ All-issues/Issue. 50. html?iid=68\&aid=9

O'Rahilly, R. and Müller, F. (1987). Developmental stages in human embryos: Including a revision of Streeter's "horizons" and a survey of the Carnegie Collection. Washington, DC, USA: Carnegie Institution of Washington, Publication No. 63. http://bit.ly/11DFONt

Opitz, R., Maquet, E., Huisken, J. et al. (2012). Transgenic zebrafish illuminate the dynamics of thyroid morphogenesis and its relationship to cardiovascular development. Dev Biol 372, 203-216. http://dx.doi.org/10.1016/j.ydbio. 2012.09.011

Pedersen, F., de Bruijn, J., Munn, S. and van Leeuwen, K. (2003). Assessment of additional testing needs under
REACH: Effects of (Q)SARS, risk based testing and voluntary industry initiatives. http://bit.ly/1iWDYIO

Piersma, A. H., Genschow, E., Verhoef, A. et al. (2004). Validation of the postimplantation rat whole-embryo culture test in the international ECVAM validation study on three in vitro embryotoxicity tests. Altern Lab Anim 32, 275-307.

Piersma, A.H. (2006). Alternative methods for developmental toxicity testing. Basic Clin Pharmacol 98, 427-431. http:// dx.doi.org/10.1111/j.1742-7843.2006.pto_373.x

Pitsiavas, V. (1999). Amiodarone compared with iodine exhibits a potent and persistent inhibitory effect on $\mathrm{TSH}-$ stimulated cAMP production in vitro: A possible mechanism to explain amiodarone-induced hypothyroidism. Eur $J$ of Endocrinol 140, 241-249. http://dx.doi.org/10.1530/ eje.0.1400241

Raldúa, D. and Babin, P. J. (2009). Simple, rapid zebrafish larva bioassay for assessing the potential of chemical pollutants and drugs to disrupt thyroid gland function. Environ Sci Technol 43, 6844-6850. http://dx.doi.org/10.1021/ es9012454

REACH (2006). Regulation (EC) No 1907/2006 of the European parliament and of the council of 18 December 2006 concerning the Registration, Evaluation, Authorisation and Restriction of Chemicals (REACH), establishing a European Chemicals Agency, amending Directive 1999/45/EC and repealing Council Regulation (EEC) No 793/93 and Commission Regulation (EC) No $1488 / 94$ as well as Council Directive 76/769/EEC and Commission Directives 91/155/ EEC, 93/67/EEC, 93/105/EC and 2000/21/EC. Off J Europ Union L396, 1-516.

Shi, Y.-B., Wong, J. and Puzianowska-Kuznicka, M. (1996). Thyroid hormone receptors: Mechanisms of transcriptional regulation and roles during frog development. J Biomed Sci 3, 307-318. http://dx.doi.org/10.1007/BF02257960

Stoker, T. E., Ferrell, J. M., Laws, S. C. et al. (2006). Evaluation of ammonium perchlorate in the endocrine disruptor screening and testing program's male pubertal protocol: ability to detect effects on thyroid endpoints. Toxicology 228, 58-65. http://dx.doi.org/10.1016/j.tox.2006.08.026

Strawson, J., Zhao, Q. and Dourson, M. (2004). Reference dose for perchlorate based on thyroid hormone change in pregnant women as the critical effect. Regul Toxicol Pharm 39, 44-65. http://dx.doi.org/10.1016/j.yrtph.2003.10.003

Strum, J. M., Wicken, J., Stanbury, J. R. et al. (1971). Appearance and function of endogenous peroxidase in fetal rat thyroid. J Cell Biol 51, 162-175. http://dx.doi.org/10.1083/ jcb.51.1.162

Tan, S. W., Timm, G. E. and Amcoff, P. (2007). History and genesis of the detailed review of thyroid hormone disruption assays. Crit Rev Toxicol 37, 1-4. http://dx.doi. org/10.1080/10408440601120855

Taurog, A. (1976). The mechanism of action of the thioureylene antithyroid drugs. Endocrinology 98, 1031-1046. http:// dx.doi.org/10.1210/endo-98-4-1031

Teixidó, E., Piqué, E., Gómez-Catalán, J. et al. (2013). Assessment of developmental delay in the zebrafish embryo 
teratogenicity assay. Toxicol In Vitro 27, 469-478. http:// dx.doi.org/10.1016/j.tiv.2012.07.010

Utiger, R. D. (1999). Maternal hypothyroidism and fetal development. $N$ Engl J Med 341, 601-602. http://dx.doi. org/10.1056/NEJM199908193410809

Valensise, H., Civitella, C., Garzetti, G. G. et al. (1992). Amiodarone treatment in pregnancy for dilatative cardiomyopathy with ventricular malignant extrasystole and normal maternal and neonatal outcome. Prenatal Diag 12, 705-708. http://dx.doi.org/10.1002/pd.1970120903

van der Jagt, K., Munn, S. J., Torslov, J. and de Bruijn, J. (2004). Alternative approaches can reduce the use of test animals under REACH. http://bit.ly/1jibqLe (accessed 31. 03.2014).

Wang, S., Aarts, J. M. M. J. G., Evers, N. M. et al. (2012). Proliferation assays for estrogenicity testing with high predictive value for the in vivo uterotrophic effect. J Steroid Biochem 128, 98-106. http://dx.doi.org/10.1016/j.jsbmb. 2011.11.009

Wendl, T., Lun, K., Mione, M. et al. (2002). pax2.1 is required for the development of thyroid follicles in zebrafish. Development 129, 3751-3760.

Wendl, T., Adzic, D., Schoenebeck, J. J. et al. (2007). Early developmental specification of the thyroid gland depends on han-expressing surrounding tissue and on FGF signals. Development 134, 2871-2879. http://dx.doi.org/10.1242/ dev.02872

Witschi, E. (1962). Development of the rat. In P. L. Altman and D. Dittmer Katz (eds.), Growth, Including Reproduction and Morphological Development (304-414). Washington DC, USA: Fed Am Soc Exp Biol.

Yamasaki, K., Tago, Y., Nagai, K. et al. (2002). Comparison of toxicity studies based on the draft protocol for the "Enhanced OECD Test Guideline no. 407" and the research protocol of "Pubertal Development and Thyroid Function in Immature Male Rats" with 6-n-propyl-2-thiouracil. Arch Toxicol 76, 495-501. http://dx.doi.org/10.1007/s00204-0020371-5

York, R. G., Funk, K. A., Girard, M. F. et al. (2003). Oral (drinking water) developmental toxicity study of ammonium perchlorate in Sprague-Dawley rats. Int J Toxicol 22, 453-464. http://dx.doi.org/10.1177/109158180302200606

York, R. G., Lewis, E., Brown, W. R. et al. (2005). Refining the effects observed in a developmental neurobehavioral study of ammonium perchlorate administered orally in drinking water to rats. I. Thyroid and reproductive effects. Int $J$ Toxicol 24, 403-418. http://dx.doi. org/10.1080/10915810500366765

Zheng, X.-M., Liu, H.-L., Shi, W. et al. (2011). Effects of perfluorinated compounds on development of zebrafish embryos. Environ Sci Pollut Res Int 19, 2498-2505. http://dx.doi. org/10.1007/s11356-012-0977-y

\section{Acknowledgements}

This project was financially supported by The Netherlands Genomics Initiative (Netherlands Toxicogenomics Centre, grant number 6162500134).

\section{Correspondence to}

Barae Jomaa, MSc

Postbus 8000

6700 EA Wageningen, Building 92

The Netherlands

Phone: +31 317482137

Fax: +31 317484931

e-mail: barae.jomaa@wur.nl 